\title{
Usages Traditionnels Et Disponibilité Des Plantes Exploitées Dans L'artisanat Chez Les Populations Koulango Et Lobi De La Périphérie Est Du Parc National De La Comoé, Côte d'Ivoire
}

\author{
Yao Bertin Kouakou, \\ Ménéké Distel Kougbo, \\ Amenan Sylvie Konan, \\ Université Nangui Abrogoua,
} U.F.R. des Sciences de la Nature, Abidjan, Côte d'Ivoire

Djah François Malan, Université Nangui Abrogoua,

U.F.R. des Sciences de la Nature, Abidjan, Côte d'Ivoire Institut Botanique Aké-Assi d'Andokoi, Abidjan, Côte d'Ivoire

\section{Adama Bakayoko,}

Université Nangui Abrogoua,

U.F.R. des Sciences de la Nature, Abidjan, Côte d'Ivoire

Centre Suisse de Recherches Scientifiques en Côte d'Ivoire,

Abidjan, Côte d'Ivoire

\section{Résumé}

Cette étude vise à faire l'inventaire et à évaluer la disponibilité des plantes utilisées dans la construction et la confection des outils par les populations Koulango et Lobi. Les enquêtes ethnobotaniques à travers les approches du «porte à porte » et des randonnées ont permis d'obtenir les informations sur les usages des plantes. La disponibilité des ressources a été évaluée à travers l'indice de raréfaction à partir des relevés de surface. Le niveau de connaissance des usages des plantes a été évalué à l'aide de la fréquence de citation. L'intensité d'utilisation des plantes a été calculée grâce à la valeur d'usage ethnobotanique. Les similitudes entre les usages des plantes ont été révélées grâce à la classification hiérarchique ascendante. Le statut des espèces a été déterminé par l'indice de raréfaction. De ces travaux, il ressort que 39 plantes appartenant à 38 genres et 22 familles sont utilisées dans la construction et 55 espèces végétales, reparties en 48 genres et 22 familles, sont utilisées dans la fabrication des objets domestiques. Les usages de six plantes sont très connus. Il s'agit de Raphia sudanica $(92,15 \%)$, 
Vitellaria paradoxa (89,6\%), Khaya senegalensis $(83,05 \%)$, Pterocarpus erinaceus $(70,15 \%)$, Phoenix reclinata $(52,75 \%)$ et Pseudocedrela kotschyi $(51,7 \%)$. Pour les deux ethnies confondues, cinq plantes sont les plus utilisées. Ce sont Raphia sudanica $(1,84)$, Vitellaria paradoxa $(1,76)$, Phoenix reclinata $(1,74)$, Khaya senegalensis $(1,66)$ et Pterocarpus erinaceus $(1,38)$. Plusieurs plantes dont Bombax costatum, Ceiba pentandra, Adansonia digitata ont des individus juvéniles et adultes très rares dans le PNC comme dans le domaine rural.

Mots-clés : Usages, Disponibilité, Parc National de la Comoé, Côte d'Ivoire

\title{
Traditional Use and Availability of Handicrafts Plants Exploit in Koulango and Lobi Populations on Eastern Periphery of Comoé National Park, Côte d'Ivoire
}

\author{
Yao Bertin Kouakou, \\ Ménéké Distel Kougbo, \\ Amenan Sylvie Konan,
} Université Nangui Abrogoua,

U.F.R. des Sciences de la Nature, Abidjan, Côte d'Ivoire

Djah François Malan,

Université Nangui Abrogoua,

U.F.R. des Sciences de la Nature, Abidjan, Côte d'Ivoire Institut Botanique Aké-Assi d'Andokoi, Abidjan, Côte d'Ivoire

Adama Bakayoko, Université Nangui Abrogoua,

U.F.R. des Sciences de la Nature, Abidjan, Côte d'Ivoire Centre Suisse de Recherches Scientifiques en Côte d'Ivoire, Abidjan, Côte d'Ivoire

\footnotetext{
Abstract

This paper focuses on keeping inventory and assessing the availability of plants used in the construction and making of tools by the Koulango and Lobi populations. Ethnobotanical surveys through door-to-door and trekking approaches have provided information on the uses of plants. The availability of resources was assessed using the scarcity index from surface surveys. The level of knowledge of plant uses was assessed using the frequency of citation.
} 
The intensity of use of the plants was calculated using the ethnobotanical use value. The similarities between the uses of plants were revealed, thanks to the ascending hierarchical classification. The status of the species was determined by the depletion index. From this work, it emerges that 39 plants belonging to 38 genera and 22 families are used in construction. 55 plant species, divided into 48 genera and 22 families, are used in the manufacture of household objects. The uses of six plants are well known. These are Raphia sudanica (92.15\%), Vitellaria paradoxa (89.6\%), Khaya senegalensis (83.05\%), Pterocarpus erinaceus (70.15\%), Phoenix reclinata (52.75\%), and Pseudocedrela kotschyi $(51.7 \%)$. For the two ethnic groups combined, five plants are the most used. These are Raphia sudanica (1.84), Vitellaria paradoxa (1.76), Phoenix reclinata (1.74), Khaya senegalensis (1.66), and Pterocarpus erinaceus (1.38). Several plants, including Bombax costatum, Ceiba pentandra, Adansonia digitata, have juvenile and adult individuals which are very rare in the PNC as in the rural area.

Keywords: Use, Availability, Comoé National Park, Côte d'Ivoire

\section{Introduction}

La flore spontanée joue un rôle très important dans l'équilibre socioéconomique des populations des pays en développement surtout des milieux ruraux (Guigma et al., 2012). En Côte d'Ivoire, depuis plusieurs millénaires, les plantes sont sollicitées pour les multiples produits et services qu'elles offrent. En effet, ces plantes sont utilisées comme nourriture (Ouattara et al., 2016) et médicaments (Béné et al., 2016). En outre, des plantes sont utilisées pour détecter avec une certaine assurance des terres fertiles (Adou Yao et al., 2016 ; Kouakou et al., 2018) et des variations de saisons (Kouakou et al., 2018). Enfin, des plantes sont utilisées comme ornements (Amani et al., 2019) et pour lutter contre les insectes et les tiques des animaux et des cultures des champs (Azokou et al., 2016). Malgré nombreuses études ethnobotaniques réalisées dans de nombreuses régions en Côte d'Ivoire, il y a peu d'informations sur les plantes utilisées dans la construction et la confection des outils en milieu rural. En plus, avec l'augmentation galopante de la population de la Région du Bounkani (Gauze \& Kanga, 2016) en particulier de la périphérie Est du Parc national de la Comoé (Kouakou et al., 2018) et face à l'interdiction de prélever et ou récolter des ressources dans le PNC il y'a urgence de faire une étude sur les plantes utilisées dans la construction et la confection des outils par les populations riveraines de ce parc. Enfin, en Côte d'Ivoire, les prélèvements se traduisent souvent sur le milieu naturel, par une pression écologique, de plus en plus lourde (Vroh et al., 2014). Ainsi, pour documenter, pérenniser cette connaissance traditionnelle et fournir des informations sur les espèces les plus utilisées, cette étude qui vise à faire 
l'inventaire des plantes utilisées dans la construction et la confection des outils par les populations de ces deux ethnies a été entreprise. L'objectif principal de l'étude est de fournir des informations sur les plantes utilisées dans le domaine de l'artisanat par les populations Koulango et Lobi de la périphérie Est du Parc national de la Comoé. De façon spécifique, d'abord, il s'agit de faire l'inventaire des usages des plantes utilisées dans le domaine de l'artisanat par les Koulango et Lobi de la périphérie Est du Parc National de la Comoé. Ensuite, recenser tous les objets fabriqués traditionnels par les Koulango et Lobi de la périphérie Est du Parc National de la Comoé. Enfin, évaluer la disponibilité de ces ressources à l'intérieur du Parc et dans le domaine rural.

\section{Site d'étude}

L'étude s'est déroulée dans 10 villages situés à la périphérie Est du Parc national de la Comoé. Ce Parc est situé dans le Nord-Est de la Côte d'Ivoire (Figure 1) entre les latitudes $8^{\circ} 30^{\prime}$ et $9^{\circ} 37^{\prime}$ Nord et les longitudes $3^{\circ} 07^{\prime}$ et $4^{\circ} 26^{\prime}$ Ouest (Gauze \& Kanga, 2016). Il couvre une superficie totale de 1 149150 hectares (OIPR, 2015). La région du Bounkani bénéficie d'un climat soudano-guinéen caractérisé par deux saisons dont une saison de pluies et une saison sèche. La saison humide dure six à sept mois (Kambiré, 2010). La saison sèche s'étend de novembre à avril avec une période d'harmattan de décembre à février (Soro et al., 2015). Le réseau hydrographique du PNC est presque entièrement constitué par le fleuve Comoé (Gauze \& Kanga, 2016). Selon ces auteurs, seule la frange orientale du Parc est drainée par des affluents de la Volta noire, rivière frontalière entre la Côte d'Ivoire et le Ghana. A ces cours d'eau, il faut ajouter de nombreuses marres (la marre Matta en est la plus importante) disséminées sur l'ensemble du Parc dont la plupart s'assèchent pendant la saison sèche (Koulibaly, 2008). 


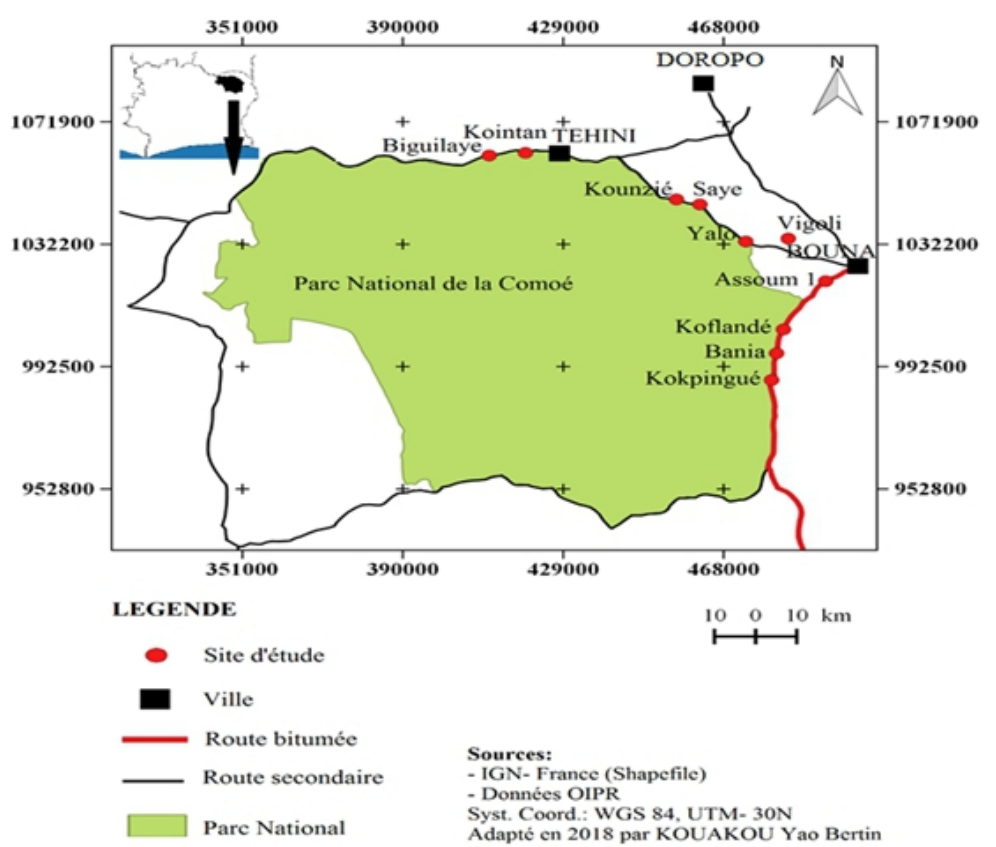

Figure 1. Localisation des sites d'étude, à la périphérie est du Parc national de la Comoé

\section{Matériel et Méthode de collecte des données}

\subsection{Matériel}

Le matériel végétal utilisé dans cette étude est constitué des espèces végétales utilisées par les populations et de l'équipement ordinaire pour faire l'inventaire floristique.

\subsection{Méthode de collecte des données}

La collecte des informations s'est tenue dans dix (10) villages. Les enquêtes ethnobotaniques ont permis d'obtenir les informations sur les plantes utilisées dans la construction et dans la fabrication des objets. Ces enquêtes ethnobotaniques se sont déroulées de septembre 2015 à décembre 2016. Les principales données collectées lors des enquêtes sont relatives à : (i)- espèces végétales collectées par les populations dans le domaine de l'artisanat (ii)parties de l'espèce végétale exploitée (iii)- nom en langue locale 'Koulango' et 'Lobi' des plantes utilisées, (iv)- importance de l'utilisation de chaque espèce végétale, (v)- le type de construction, (vi)- la partie de la construction, (vii)- le type d'outils fabriqués. Les questions ont été posées en français et traduit dans la langue locale : Koulango ou Lobi par le guide-interprète. Le guide a été choisi sur la base de sa bonne connaissance des plantes dans sa langue locale.

Deux méthodes ont été utilisées. La méthode du « porte à porte »a consisté à interviewer la population de foyer en foyer. Pour ce faire, l'approche de la liste ouverte ou « free-listing » a été utilisée. Lors de son entrevue à l'aide 
d'un questionnaire, le répondant est invité à citer les espèces utilisées qu'il connaît. Cette approche est particulièrement adaptée aux études ethnobotaniques utilisant les réponses spontanées (Thompson \& Zhang, 2006). Elle repose sur le principe que les plantes employées les plus significatives sont mentionnées par plusieurs informateurs et obtiennent ainsi un rang élevé (Malan, 2008). Après les entretiens avec la méthode du «porte à porte », quelques visites dans la végétation environnante avec certains informateurs ou avec un guide à l'aide de la méthode des randonnées ont été effectuées. Ces randonnées, appelées également la méthode du « walk-in-thewoods» ont pour premier objectif de collecter les ressources végétales utilisées par l'enquêté (Thomas et al., 2009). Le second objectif de cette méthode est de connaitre les plantes, leurs noms dans les langues locales, leurs usages, les parties utilisées et faire des études comparatives (Thomas et al., 2009). En outre, cette méthode permet de se familiariser avec la végétation et, surtout, de constituer un herbier pour l'identification des échantillons au laboratoire.

\subsection{Identification des plantes}

L'identification des échantillons a été faite avec les ouvrages suivants : le logiciel Ligneux du sahel V.1.0. (Bonnet et al., 2005), Aké-Assi (2001), Aké Assi (2002). La confirmation de ces identifications a été faite en référence à l'herbier national du Centre National de Floristique (CNF) de l'Université Félix Houphouet-Boigny de Cocody. La classification des familles suit la quatrième version du Groupe pour la Phylogénie des Angiospermes (APG IV, 2016) et les noms scientifiques ont été actualisés grâce à la base de données APD (African Plants Database version 3.4.0).

\subsection{Collecte des données de la disponibilité des plantes}

La disponibilité des ressources a été évaluée à travers l'indice de raréfaction à partir des relevés de surface. Pour ce faire, 10 placettes de $30 \mathrm{~m}$ x $30 \mathrm{~m}$, soit une superficie de $900 \mathrm{~m} 2$ chacune, ont été installées par intervalle de $500 \mathrm{~m}$ (Figure 2). Ces placettes ont été installées dans les différents faciès floristiques des terres cultivables des différents villages et 10 autres placettes en sens opposé dans le PNC. Au total, 200 placettes ont été installées. Au sein de ces placettes, la présence des espèces alimentaires a été notée.

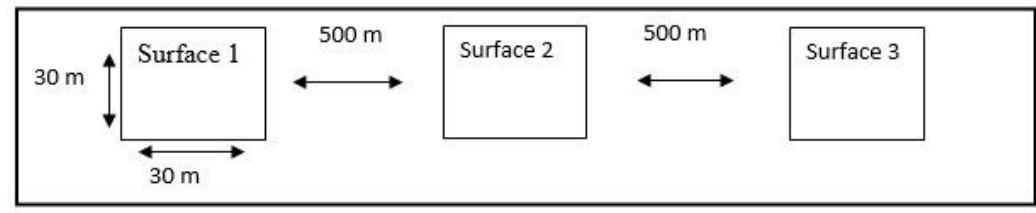

Figure 2. Schéma du dispositif expérimental pour étude de la disponibilité des ressources 


\subsection{Traitement des données}

\subsubsection{Niveau de connaissance des usages des plantes}

Le niveau de connaissance des usages des plantes est traduit par la fréquence de citation de chaque espèce végétale (Malan, 2008). Cette fréquence de citation est le nombre de fois qu'une plante est citée par un interlocuteur. C'est un indice basé sur l'usage consensuel des plantes qui permet d'estimer la crédibilité des informations reçues (Schrauf \& Sanchez, 2008). La fréquence de citation d'une espèce (FC) est calculée par la formule de Grangé \& Lebar (1993):

Où :

$$
\mathrm{FC}=\frac{\mathrm{n}}{\mathrm{N}} \times 100
$$

$\mathrm{n}$ : est le nombre de personnes interrogées ayant cité la plante ; $\mathrm{N}$ est le nombre total de personnes interrogées au cours de l'enquête dans une zone donnée. Les espèces sont alors reparties selon les classes suivantes (Kouamé et al., 2008) : une espèce est dite bien connue si le niveau de connaissance est compris entre $50 \%$ et $100 \%$; une espèce est dite moyennement connue si le niveau de connaissance est compris entre $25 \%$ et $50 \%$; une espèce est dite peu connue si le niveau de connaissance est compris entre $00 \%$ et $25 \%$.

\subsubsection{Intensité d'utilisation des plantes}

La valeur d'usage ethnobotanique d'une espèce représente l'intensité d'utilisation de cette espèce par les communautés (Tiétiambou et al., 2016). La valeur d'usage ethnobotanique (VUi) d'une espèce au sein d'une catégorie d'usage est représentée par son score moyen d'utilisation au sein de la catégorie d'usage. Selon Martin (1995), l'application des méthodes ethnobotaniques utilisant des scores permettent de cerner l'importance accordée à des plantes par des populations locales. Leur intérêt réside dans le fait qu'elles permettent de déterminer de façon significative les espèces ayant une grande importance au sein de la population. L'exploitation des valeurs d'usage se révèle être un outil de base dans la sélection des espèces d'intérêt socio-économique, culturel et objet de forte pression anthropique (Dossou et al., 2012). La valeur d'usage ethnobotanique unitaire (VUi) a été calculée pour toutes les espèces végétales et dans toutes les catégories suivant la méthode de calcul utilisée par Belem et al. (2008).

La valeur d'usage d'une espèce donnée $(\mathrm{k})$ au sein d'une catégorie d'usage donnée est représentée par son score moyen d'utilisation au sein de cette catégorie. Elle est calculée par la formule suivante utilisée par Philips \& Gentry (1993):

Où,

$$
\mathrm{VU}(\mathrm{k})=\frac{\sum_{\mathrm{i}}^{\mathrm{n}} \mathrm{Sj}}{\mathrm{N}}
$$


VU(k) est la valeur d'usage ethnobotanique de l'espèce $\mathrm{k}$ au sein d'une catégorie d'usage donnée ; $\mathrm{Sj}$ est le score d'utilisation attribué par le répondant j; $\mathrm{N}$ est le nombre de répondants pour une catégorie d'usage donnée.

La grille d'appréciation utilisée dans cette étude par les informateurs est :

- 2 = espèce fortement utilisée ;

- 1 = espèce faiblement utilisée.

Dans cette étude, les espèces sont reparties dans trois groupes : une espèce est dite très utilisée lorsque sa valeur d'usage est comprise entre 1,2 et 2 ; une espèce est dite moyennement utilisée lorsque sa valeur d'usage est comprise entre 0,66 et 1,2 ; une espèce est dite faiblement utilisée lorsque sa valeur d'usage est comprise entre 0 et 0,66 .

\subsubsection{Similitudes des usages des plantes chez les populations Koulango et Lobi}

Les similitudes entre les usages des plantes ont été révélées grâce à la classification hiérarchique ascendante $(\mathrm{CAH})$. Cette classification a pour principal objectif de rassembler les éléments qui se ressemblent et /ou de séparer ceux qui diffèrent. Elle consiste à construire une succession de partitions emboîtées par regroupements successifs des observations en classes de moins en moins fines, jusqu'à l'obtention d'une seule classe contenant tous les objets (Ouattara, 2014). Parmi les méthodes de regroupement de classes utilisées en classification hiérarchique, c'est la méthode de Ward qui a été utilisée (Ward, 1963). Elle est basée sur la minimisation de la variance intraclasse (Chimi Chiadjeu et al., 2016). Parmi les distances utilisées pour la CAH, c'est la distance euclidienne qui a été choisie. Ces analyses ont été réalisées avec l'environnement statistique $\mathrm{R}$ version 3.5.1 (R Development Core Team, 2016).

\subsubsection{Statut des espèces}

Le statut des espèces a été déterminé à l'aide l'indice de raréfaction calculé à partir de l'équation de Géhu \& Géhu (1980). Cette méthode, largement utilisée pour l'évaluation de la disponibilité des ressources végétales en Afrique (Piba et al., 2015), consiste à comparer deux communautés sur la base de la diversité obtenue pour un échantillon de taille identique. Pour la présente étude, la mesure des dbh s'est faite à 1,30 m du sol (Dro et al., 2013 ; Vroh Bi, 2013). Dans le cas de certains individus, dont des branches se ramifient à une hauteur inférieure à $1,30 \mathrm{~m}$, la mesure des dbh a été faite à la base à $30 \mathrm{~cm}$ au-dessus du sol (Thiombiano et al., 2010). Les rubans gradués de $50 \mathrm{~m}$ ont permis de mesurer les contours des aires de relevés. La circonférence de $10 \mathrm{~cm}$ soit un $\mathrm{dbh} \geq 3,18 \mathrm{~cm}$ a été retenue, car, cela permet de prendre en compte un nombre plus important d'individus, dans une zone composée principalement de savanes. Dans le traitement des données, le terme 
de juvéniles est donné aux plantes dont les circonférences sont inférieures à $10 \mathrm{~cm}(\mathrm{dbh}<3,18 \mathrm{~cm})$ et celui d'adultes à celles dont les circonférences sont supérieures à $10 \mathrm{~cm}(\mathrm{dbh} \geq 3,18 \mathrm{~cm})$. Cet indice se calcule selon l'équation suivante Géhu \& Géhu (1980) :

$R i=\left(1-\frac{n i}{N}\right) X 100$

avec, $R i$ l'indice de raréfaction de l'espèce $i$, $n i$ le nombre de placettes où l'espèce $i$ est retrouvée et $N$ le nombre total de placettes posées dans le milieu. Cet indice varie de 0 à 100 et se répartit en six classes de valeur (Tableau II). Ainsi, par exemple, une espèce dont l'indice de raréfaction est compris entre 80 et $100 \%$ témoigne de la rareté de cette ressource.

Tableau I. Interprétation des valeurs de l'indice de raréfaction

Classes des fréquences $(\%)$

\begin{tabular}{cc}
\hline $\mathbf{R i}<\mathbf{1 0}$ & Espèce très abondantes \\
\hline $\mathbf{1 0} \leq \mathbf{R i}<\mathbf{2 0}$ & Espèce fréquente \\
$\mathbf{2 0} \leq \mathbf{R i}<\mathbf{4 0}$ & Espèce constante \\
\hline $\mathbf{4 0} \leq \mathbf{R i}<\mathbf{6 0}$ & Espèce accessoire \\
\hline $\mathbf{6 0} \leq \mathbf{R i}<\mathbf{8 0}$ & Espèce accidentelle ou rare \\
$\mathbf{8 0} \leq \mathbf{R i} \leq \mathbf{1 0 0}$ & Espèce très rare ou menacée dans la \\
& région
\end{tabular}

\section{Resultats}

3.1. Plantes utilisées dans la construction des habitations humaines

De ces travaux, il ressort que 23 espèces végétales sont utilisées dans la zone pour la construction. Ces espèces sont recherchées principalement pour leur tige ou pseudo tronc. Généralement, la toiture des constructions est faite avec des plantes avant que celle-ci ne soit couverte avec Andropogon gayanus (Figures 3).

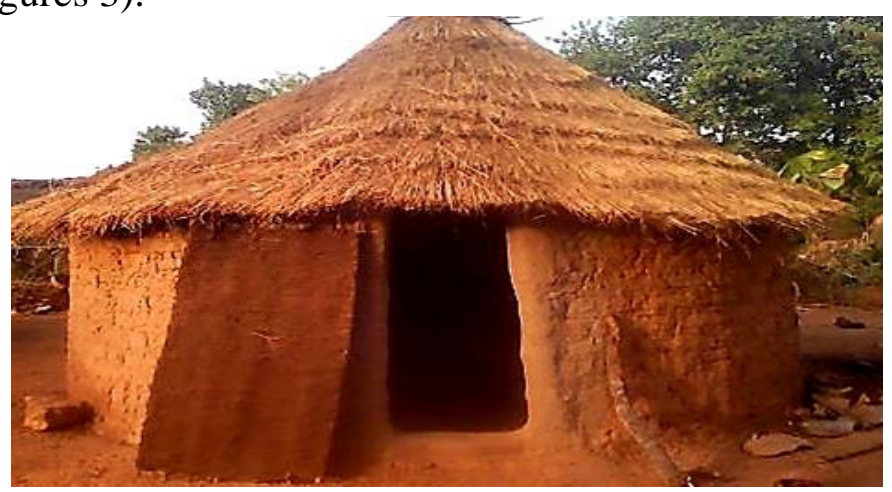

Figure 3. Case ronde à toiture conique couverte par Andropogon gayanus chez les Koulango et les Lob

\subsection{Plantes utilisées dans les autres types de construction}

Dans ce groupe sont classées les végétaux ou produits de végétaux utilisés pour construire : les cuisines, les hangars et les greniers. Vingt et une espèces végétales sont utilisées comme traverse des cuisines, greniers et des 
hangars (Vitellaria paradoxa, Detarium microcarpum, Raphia sudanica, Terminalia avicennioides, Uapaca togoensis, etc.). Six espèces végétales peuvent être utilisées comme fourches des cuisines, des hangars et des greniers de maïs. Ce sont: Hymenocardi acida, Lophira lanceolata, Diospyros mespiliformis, Terminalia avicennioides, Vitellaria paradoxa et Uapaca togoensis. Les toitures des cuisines, des greniers sont généralement couvertes avec Andropogon gayanus (Figure 4).

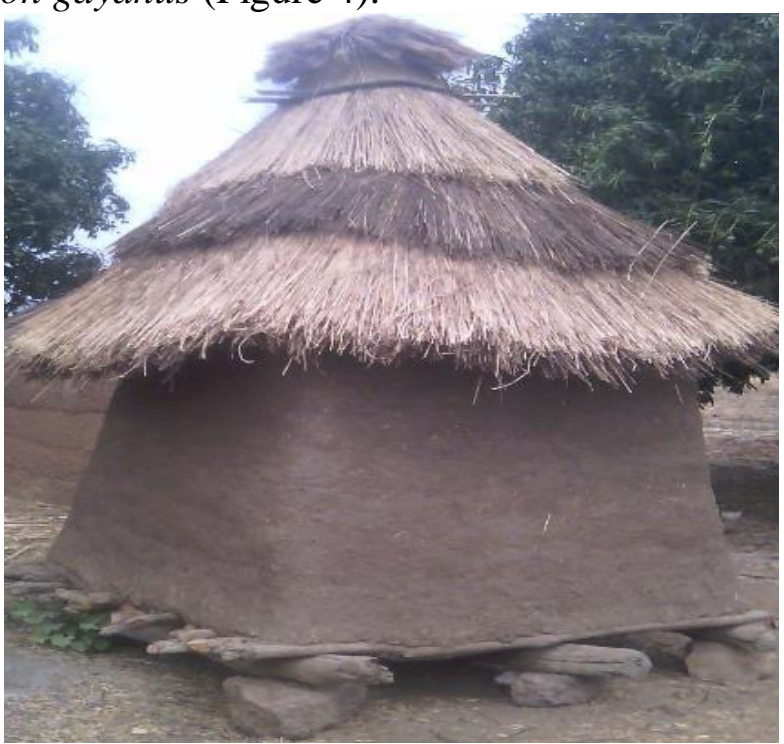

Figure 4. Grenier de mil et de sorgho à toiture conique couvert avec Andropogon gayanus chez les Lobi

\subsection{Plantes utilisées dans la fabrication des objets domestiques \\ $\checkmark$ Plantes utilisées dans la fabrication des ustensiles de cuisine}

Les ustensiles de cuisine fabriqués dans la zone sont : les mortiers (Figure 5), les pilons, les spatules et les paniers. Plusieurs plantes dont Vitellaria paradoxa fournissent les meilleurs bois pour tailler les mortiers et les pilons. Généralement, les paniers sont faits avec les rachis et les folioles de Elaeis guineensis et de Raphia sudanica. Dans les localités où il y a la disparition ou l'absence de ces deux essences de valeur, les populations ont mis sur place un savoir-faire local qui leur a permis d'exploiter de manière efficace leur environnement végétal. Par exemple à Biguilaye, les paniers sont faits avec Landolphia heudelotii, à Yalo avec Saba senegalensis (Figures 6). Une plante (Bombax costatum), sert à fabriquer les spatules. 


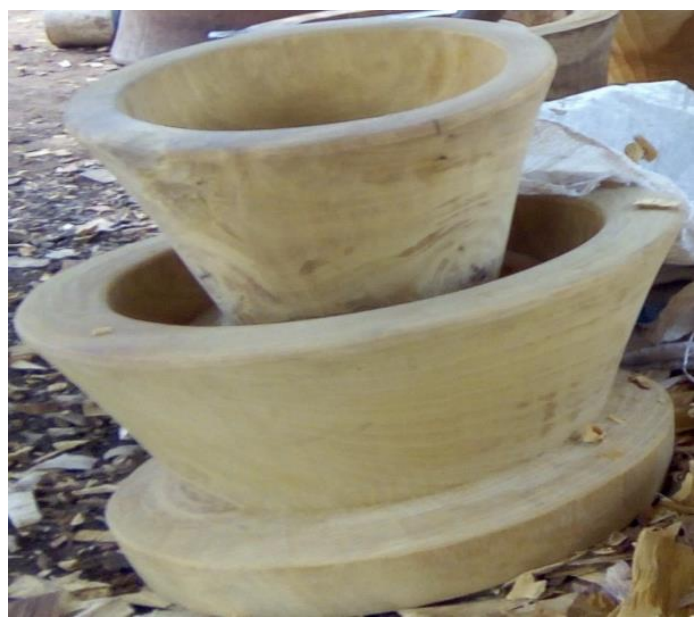

Figure 5. Mortiers fabriqués avec les tiges de Manilkara multinervis chez les Koulango et les Lobi

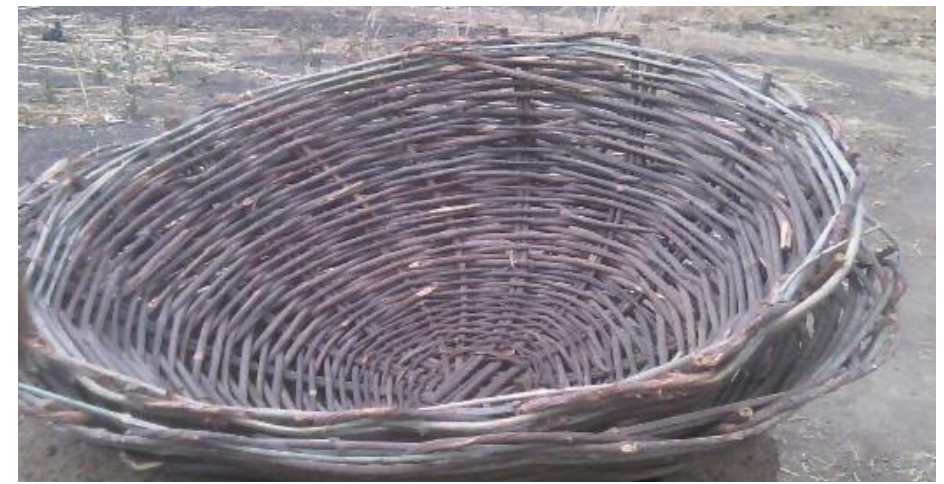

Figure 6. Panier fabriqué avec les tiges de Landolphia heudelotii chez les Koulango et les Lobi

\section{$\checkmark$ Plantes utilisées dans la fabrication des mobiliers}

Les mobiliers fabriqués par les Koulango et les Lobi sont : les lits, les tables, les chaises et les tabourets. Quatre espèces végétales sont utilisées pour fabriquer les chaises traditionnelles. Ce sont : Raphia sudanica, Mitragyna inermis, Azadirachta indica et Hannoa undulata: Les tabourets présentent une grande diversité. Certains sont sculptés, d'autres ne le sont pas. Ils sont aussi tirés des bois les plus variés. Les bois durs, légers et faciles à tailler et les bois tendres se mélangent comme si cet instrument de la convivialité ne voulait déplaire à aucune espèce végétale. Le tronc de Bombax costatum et celui de Ceiba pentandra sont utilisés pour sculpter les tabourets (Figure 7). 


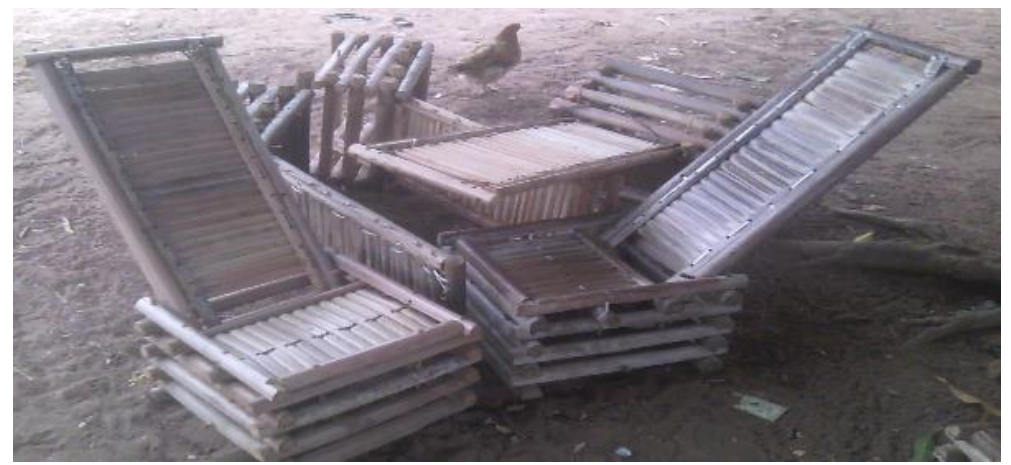

Figure 7. Rachis de Raphia sudanica utilisés dans la confection de chaises chez les Koulango et les Lobi

\section{$\checkmark$ Plantes utilisées dans la fabrication des objets aratoires}

Les objets aratoires fabriqués sont : les manches de houes et de haches. Les manches des objets aratoires sont principalement fabriquées avec les bois durs, légers et résistants aux insectes. Prosopis africana, Acacia dudgeoni, Detarium microcarpum, Khaya senegalensis, Pseudocedrela kotschyi, Manilkara multinervis, Vitellaria paradoxa fournissent les meilleurs bois pour la fabrication des manches des objets aratoires (Figure 8).

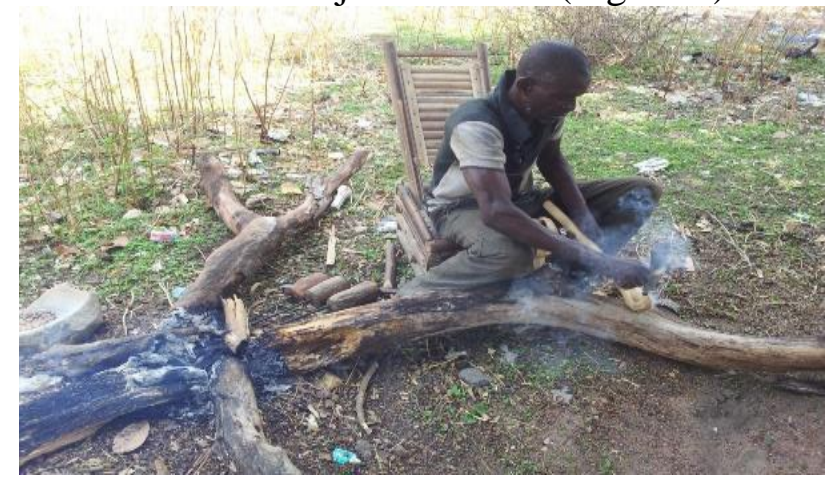

Figure 8. Fabrication d'une manche de houe chez les Koulango

\section{$\checkmark$ Plantes utilisées dans la fabrication des objets ludiques}

Concernant les objets ludiques, les balafons (Figure 9) sont fabriqués avec généralement deux plantes (Pterocarpus erinaceus et Uapaca togoensis) mais Berlinia grandiflora est une plante utilisée temporairement. Les tamstams sont le plus souvent taillés avec Pterocarpus erinaceus et Uapaca togoensis. 


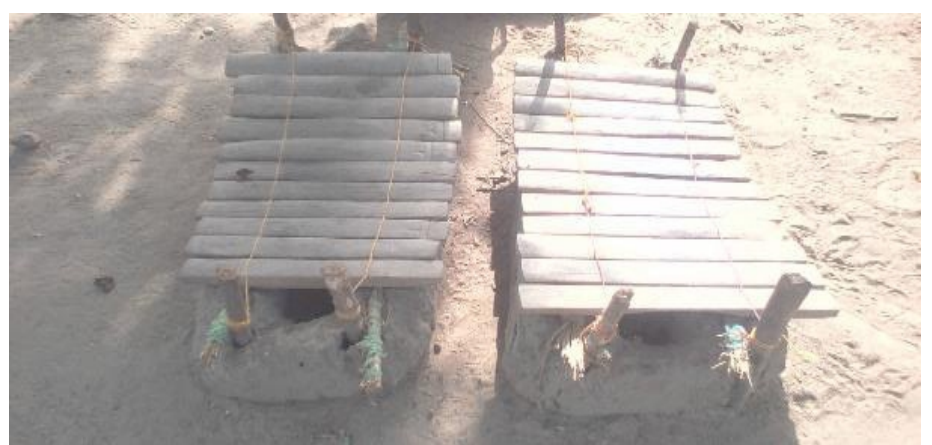

Figure 9. Balafons taillés avec les tiges de Pterocarpus erinaceus chez les Lobi

\section{$\checkmark$ Niveau de connaissance des usages des plantes par les Koulango et les Lobi}

Sur les 66 plantes recensées dans le domaine artisanal, six sont très connues. Il s'agit de Raphia sudanica (92,15\%), Vitellaria paradoxa (89,6\%), Khaya senegalensis (83,05\%), Pterocarpus erinaceus (70,15\%), Phoenix reclinata $(52,75 \%)$ et Pseudocedrela kotschyi $(51,7 \%)$. Les plantes les plus connues varient lorsque chaque ethnie est prise séparément. Chez les Koulango, sept espèces végétales sont les plus citées avec des fréquences de citation variant de $61,8 \%$ à $88,7 \%$. Ce sont : Raphia sudanica $(88,7 \%)$, Vitellaria paradoxa (86,1\%), Khaya senegalensis (75,5\%), Lophira lanceolata (70\%), Pseudocedrela kotschyi (68,6\%), Bombax costatum $(62,9 \%)$ et Phoenix reclinata $(61,8 \%)$. Chez les Lobi, par contre, ce sont quatre plantes qui sont très connues. Il s'agit de Raphia sudanica (95,6\%), Vitellaria paradoxa $(93,1 \%)$, Khaya senegalensis $(90,6 \%)$ et Pterocarpus erinaceus $(83,4 \%)$.

\subsection{Intensité d'utilisation des plantes par les Koulango et les Lobi}

Pour les deux ethnies confondues, cinq plantes sont les plus utilisées. Ce sont Raphia sudanica $(1,84)$, Vitellaria paradoxa $(1,76)$, Phoenix reclinata $(1,74)$, Khaya senegalensis $(1,66)$ et Pterocarpus erinaceus $(1,38)$. Chez les Koulango, les espèces végétales qui ont une forte intensité d'utilisation sont : Raphia sudanica $(1,77)$, Vitellaria paradoxa $(1,66)$, Khaya senegalensis $(1,59)$, Pseudocedrela kotschyi $(1,36)$, Lophira lanceolata (1,35), Bombax costatum $(1,26)$ et Phoenix reclinata $(1,22)$. Chez les Lobi, trois plantes sont fortement utilisées. Il s'agit de Raphia sudanica (1,91), Vitellaria paradoxa $(1,86)$, Khaya senegalensis $(1,72)$ et Pterocarpus erinaceus $(1,67)$.

\subsection{Similitudes des usages des plantes utilisées dans l'artisanat}

L'étude révèle qu'il y a des similitudes et des différences au niveau des usages des plantes utilisées dans le domaine de l'artisanat dans les différents villages (Figure 10). La classification ascendante hiérarchique dispose de trois 
blocs. Le bloc 1 comprend 6 villages Lobi (Saye, Kounzié, Kokpingué, Koflandé, Yalo et Bania). Le bloc 2 est également composé de 6 villages Koulango (Assoum 1, Kounzié, Yalo, Bania, Kokpingué et Saye). Les usages des plantes utilisées dans le domaine de l'artisanat sont semblables dans ces villages. Les plantes sont utilisées pour fabriquer les mêmes objets domestiques. Le bloc 3 est composé de 4 villages (2 villages Lobi et 2 villages Koulango).
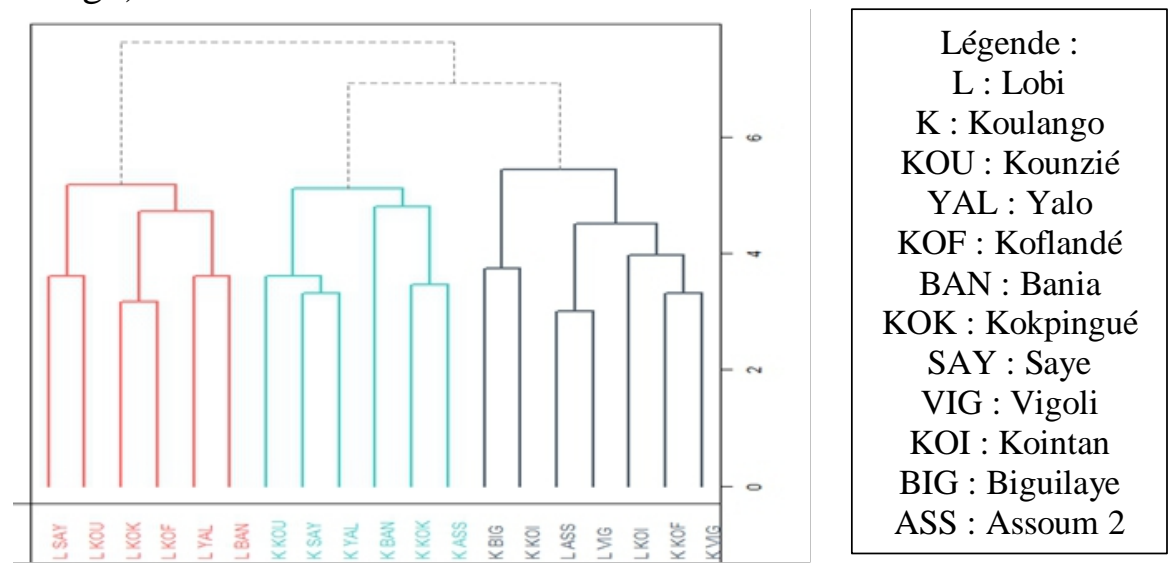

Villages visités

Figure 10. Comparaison intra et interculturelle des usages des plantes utilisées dans le domaine de l'artisanat par les populations Koulango et Lobi.

\subsection{Disponibilité des plantes utilisées par les populations Koulango et Lobi \\ $\checkmark$ Disponibilité des individus juvéniles des plantes}

Les relevés effectués dans le PNC montrent que 12 plantes (soit $18,18 \%$ ) ont des individus juvéniles "abondants". Parmi ces plantes, nous avons : Acacia dudgeonii, Pericopsis laxiflora, Terminalia avicennioides et Vitellaria paradoxa. Toutefois, il a été relevé 23 espèces soit $(38,85 \%)$ "très rares". C'est le cas de Adansonia digitata, Bombax costatum et Raphia sudanica.

Au niveau des relevés réalisés dans le domaine rural, huit plantes (soit $12,12 \%)$ ont des individus juvéniles "abondants". C'est le cas des individus juvéniles de Burkea africana, Daniellia oliveri, Hymenocardia acida, Isoberlinia doka, Terminalia avicennioides et Vitellaria paradoxa. Hormis ces plantes dont les individus juvéniles sont abondants, il a été relevé que 30 espèces (soit 45,45\%) sont "très rares". Parmi ces espèces dont les individus juvéniles sont "très rares", nous avons : Adansonia digitata, Ceiba pentandra, Cussonia barteri (Figure 11). 


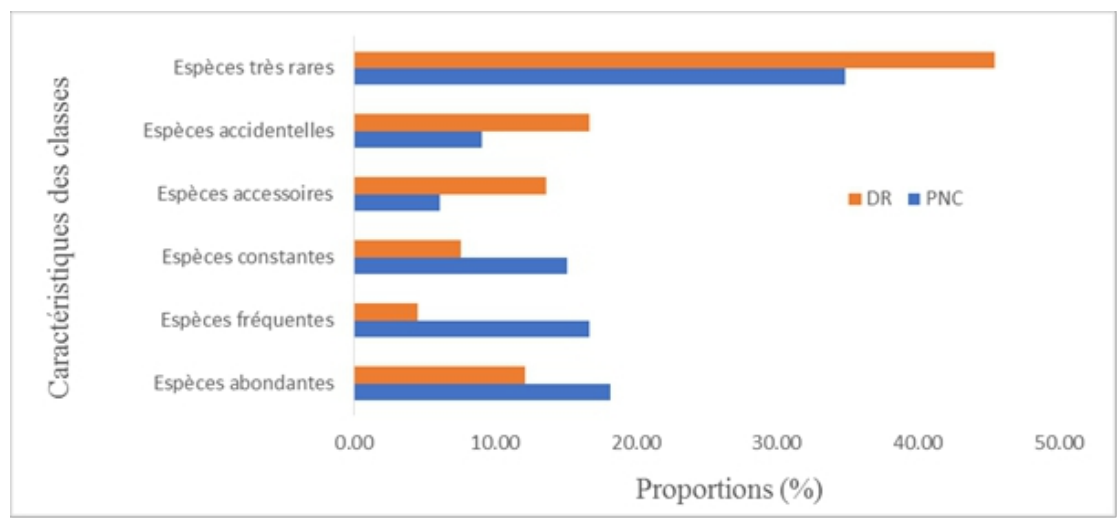

Figure 11. Disponibilité des individus juvéniles des plantes

\section{Disponibilité des individus adultes des plantes}

L'inventaire des individus adultes effectué dans le PNC et dans le domaine rural dans le cadre de cette étude révèle les résultats ci-dessous (Figure 12). Dans le PNC, les individus adultes de 13 plantes (soit 19,70\%) sont "abondants". C'est le cas des individus adultes de Daniellia oliveri, Hymenocardia acida, Isoberlinia doka, Terminalia avicennioides et Vitellaria paradoxa. Il ressort que 16 plantes (soit 24,24\%) ont des individus adultes "très rares". Parmi ces plantes, nous avons Adansonia digitata, Anogeissus leiocarpus, Bombax costatum, Ceiba pentandra, Flacourtia indica et Phoenix reclinata. Cette étude montre également que dans le domaine rural, les individus adultes de 9 plantes soit $(13,64 \%)$ sont "abondants". Parmi ces plantes, nous avons : Annona senegalensis, Saba senegalensis et Vitellaria paradoxa. Les individus adultes de Pericopsis laxiflora et de Pseudocedrela kotschyi sont "fréquents". L'étude a aussi révélé que 23 plantes dont Acacia dudgeonii, Burkea africana, Daniellia oliveri, Detarium microcarpum, Hymenocardia acida, Isoberlinia doka et Terminalia avicennioides sont "très rares".

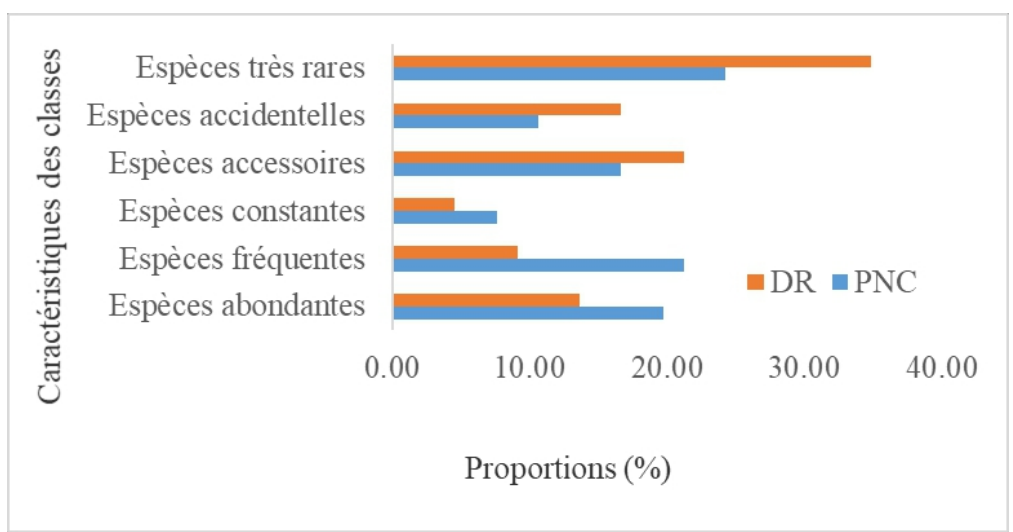

Figure 12. Disponibilité des individus adultes des plantes 


\section{Discussion}

La case des Koulango et Lobi reflète donc le paysage végétal local. Fruit d'une combinaison harmonieuse de matériaux ligneux et herbacés d'une grande hétérogénéité, ce modèle d'habitation est la traduction concrète du savoir-faire des populations locales dans la maîtrise des ressources de leur milieu naturel. Ces observations ont été également faites par plusieurs auteurs dont Ganaba et al. (2004), Biloso \& Lejoly (2006). Les Koulango et les Lobi font une sélection de plantes dans la construction. En effet, la construction doit obéir aux propriétés intrinsèques de la plante utilisée. Les critères de sélection des plantes ou des parties des plantes sont les suivants : la solidité ; la durabilité ; la résistance aux termites et aux insectes foreurs du bois ; la configuration $\mathrm{du}$ bois (droiture $\mathrm{du}$ port, présence de fourches) et sa maniabilité. Ces remarques ont été également faites par Gormo \& Nizesete (2013), Shango (2010).

Au niveau des objets domestiques, diverses plantes sont recherchées. Toutefois, les populations locales des deux ethnies (Koulango et Lobi) de tous les villages font une sélection de plantes en fonction de l'objet désiré. Ces résultats se justifieraient par le fait que le choix des espèces végétales utilisées en artisanat est souvent guidé par la valeur et l'importance sociales de l'objet fabriqué. En effet, un objet d'intérêt majeur est confectionné à partir d'un bois dur et résistant alors qu'un simple morceau de tronc d'arbre peut valablement servir à confectionner un objet commun d'importance moindre (Tiébré et al., 2016). Lophira lanceolata est une des espèces végétales utilisées par les Koulango et les Lobi pour la fabrication des pilons et des mortiers. Ce résultat pourrait s'expliquer par le fait que le bois de cette plante est solide ainsi, l'objet fabriqué peut être utilisé sur plusieurs années. Ces résultats sont semblables à ceux de Nonviho (2015). Selon les informateurs Koulango et Lobi, Khaya senegalensis est recherchée dans cette catégorie pour fabriquer des mortiers et des pilons. Boyd et al. (2014), ont rapporté l'usage de cet arbre dans la fabrication de mortier. Concernant la fabrication des outils aratoire, différentes parties de Khaya senegalensis permettent de fabriquer des manches de houe. Le choix de cette espèce pourrait se justifier par le fait que son bois serait solide et facile à modeler. Cette plante est utilisée pour fabriquer plusieurs autres objets. En effet, selon Boyd et al. (2014), Khaya senegalensis est utilisée pour la fabrication de Bancs et de chaises. La confection des objets ludiques (balafons) se fait essentiellement avec Pterocarpus erinaceus. Ce résultat se justifierait par deux raisons majeures. Premièrement, cette plante fournit à la fois du bois léger, facile à modeler et résistant aux insectes. Secondairement, une fois taillée et séché, la planche de cette plante est capable de fournir des sons variés. Cette espèce est très recherchée par les artisans Burkinabè pour la confection d'instruments de musique tels que le balafon et le djembé (Rabiou et al., 2015). 
Concernant le niveau de connaissances des usages et l'intensité d'utilisation des plantes, il faut dire que les plantes de cette catégorie sont regroupées en trois classes. La première classe comprend les plantes fortement citées et très utilisées (Pterocarpus erinaceus, Raphia sudanica, Vitellaria paradoxa, etc.). Les plantes fortement citées et faiblement utilisées sont rangées dans la $2^{\mathrm{e}}$ classe. La $3^{\mathrm{e}}$ classe regroupe les plantes faiblement citées et faiblement utilisées (Lannea barteri, Sarcocephalus latifolius, Syzygium guineense). Ces nombreuses classes du niveau de connaissances des usages et de l'intensité d'utilisation des plantes indiquent que les plantes artisanales sont choisies et utilisées en fonction des rôles que joue chaque plante. Ces remarques ont été aussi observées au Cameroun (Gormo \& Nizesete, 2013) et au Burkina Faso (Boyd et al., 2014). La faible fréquence de citation de certaines plantes utilisées pour la fabrication des chaises traditionnelles et dans la construction des maisons s'expliquerait par la concurrence des produits sur le marché. En effet, il y a des chaises plus confortables et plus résistantes fabriquées par des menuisiers professionnels. En outre, avec l'évolution, les maisons sont de plus en plus construites avec du matériel moderne.

Concernant le statut des plantes, les investigations révèlent que plusieurs plantes sont rares dans le PNC comme dans le domaine rural. Cependant, il y a plus de plantes très rares dans le domaine rural que dans le PNC. En plus, il y a moins de plantes abondantes dans le domaine rural que dans le PNC. De toute évidence, les pratiques culturales qui consistent au défrichement de nouvelles terres pour la mise en place de cultures exigeantes telles que l'igname pourraient expliquer cette différence, comme l'ont déjà fait remarquer d'autres auteurs (Zerbo et al., 2014 ; Thiombiano et al., 2016). En plus, une demande croissante des populations en bois pour la fabrication des objets (Faye, 2010) est à incriminer. En effet, la fabrication des objets domestiques exige l'utilisation des tiges par conséquent l'abattage de l'arbre. Ces coupes concernent généralement les plantes adultes. En effet, les mauvaises techniques de récolte des plantes ligneuses peuvent aussi être à l'origine de raréfaction de certaines d'entre elles telles que Khaya senegalensis, Lophira lanceolata et Manilkara multinervis. Plusieurs plantes (Bombax costatum, Ceiba pentandra, Adansonia digitata, Cussonia barteri, etc.) ont des individus juvéniles très rares dans le PNC comme dans le domaine rural. Ce résultat pourrait traduire une réelle menace sur l'avenir de ces plantes. En effet, les individus adultes sont utilisés. De ces travaux, il ressort que certaines espèces sont abondantes dans le domaine rural comme dans le PNC. Ce résultat pourrait se justifier par la protection de ces arbres. En effet, il est interdit, par exemple, aux autochtones de couper Vitellaria paradoxa. De même, selon Dubut (2012), sur le continent Africain, Vitellaria paradoxa (le karité) est sacré, de ce fait, cet arbre ne peut être coupé ni détruit. 


\section{Conclusion}

Les populations Koulango et Lobi utilisent 66 espèces de plantes dans le domaine artisanal. Quinze plantes sont utilisées uniquement par les Koulango. Alors que Entada africana est la seule plante typique aux Lobi. Les Koulango et les Lobi utilisent 39 plantes pour construire et 55 pour confectionner les objets domestiques. Les critères pour qu'une plante soit utile dans la construction dépendent du type de construction (habitation ou structure de stockage) et de la partie de la construction (poutre, traverse, charpente, etc.). Dans la fabrication des objets domestiques, les plantes sont recherchées selon l'objet à fabriquer (ustensiles de cuisine, mobilier ou objets ludiques, etc.). Ainsi, l'intensité d'utilisation d'une plante est fonction du nombre d'objet fabriqué et de la qualité intrinsèque du bois de cette plante. Les Koulango et les Lobi de certains villages utilisent les mêmes plantes pour construire ou pour fabriquer les mêmes objets domestiques. La raréfaction de plusieurs plantes dans le PNC comme dans le domaine rural s'expliquerait par les actions anthropiques et l'appartenance de la zone (six à sept mois de sécheresse). Pour une utilisation rationnelle et efficace des plantes, il faut domestiquer et introduire les plants de certaines espèces devenues rares. Il faut également promouvoir l'agroforesterie. Enfin, il faut sensibiliser les populations sur les techniques de prélèvement et la quantité de produits à récolter.

\section{References:}

1. Adou Yao, Y. C., Kpangui K. B., Vroh Bi T. A. \& Ouattara D. (2016). Pratiques culturales, valeurs d'usage et perception des paysans des espèces compagnes du cacaoyer dans des agroforêts traditionnelles au centre de la Côte d'Ivoire. Revue d'ethnoécologie, 9 : 1 - 20.

2. Aké Assi, L. (2001). Flore de la côte d'Ivoire. Catalogue systématique, biogéographie et écologie, tome I, Boissiera 57, Conservatoire et Jardin Botanique de Genève, Genève, Suisse, 396 p.

3. Aké Assi, L. (2002). Flore de la côte d'Ivoire. Catalogue systématique, biogéographie et écologie, tome II, Boissiera 58, Conservatoire et Jardin Botanique de Genève, Genève, Suisse, $401 \mathrm{p}$.

4. Amani, K. D. S. S., Kouassi, A. F. \& Aké-Assi, A. E. (2019). Diversité floristique des plantes à potentialité décorative issues des formations naturelles du Nord de la Côte d'Ivoire. European Scientific Journal, 15 (15) : $46-63$.

5. APD, base de données des plantes d'Afrique (version 3.4.0). Conservatoire et Jardin botaniques de la Ville de Genève et South African National Biodiversity Institute, Pretoria, consulté le 20/10/2018, de < http://www.ville-ge.ch/musinfo/bd/cjb/africa/>. 
6. APG (2016). An update of the Angiosperm phylogeny Group classification for the orders and families of flowering plants. Botanical Journal of the Linnean Society, 181 (1): 1 -20.

7. Azokou, A., Achi, Y. L., Koné, M. W. (2016). Lutte contre les tiques du bétail en Côte d'Ivoire par des méthodes traditionnelles. Livestock Research for Rural Development, 28 (52). URL:http://www.lrrd.org/lrrd28/4/azok280 52.htm.

8. Belem, B. (2009). Ethnobotanique et conservation de Bombax costatum Pel. \& Vuil. (faux Kapokier) dans les systèmes de production agricoles du plateau central, Burkina Faso. Thèse de Doctorat, Université de Ouagadougou, 189 p.

9. Béné, K., Camara, D., Fofié, N. B. Y., Kanga, Y., Yapi, A. B., Yapo, Y. C., Ambé, S. A. \& Zirihi, G. N. (2016). Étude ethnobotanique des plantes médicinales utilisées dans le Département de Transua, District du Zanzan (Côte d'Ivoire). Journal of Animal \& Plant Sciences, 27 : $4230-4250$.

10. Biloso, A. \& Lejoly J. (2006). Etude de l'exploitation et du marché des produits forestiers non ligneux à Kinshasa. Tropicultura, 24 (3) : 183 188.

11. Bonnet, P., Arbonnier, M. \& Grard, P. (2005). Ligneux du Sahel. Cirad - CD-Rom.

12. Boyd, R., Fournier, A. \& Nignan S. (2014). Une base de données informatisée transdisciplinaire de la flore : un outil pour l'étude du lien nature-société. Gwenaëlle Fabre, Anne Fournier, Lamine Sanogo. Regards scientifiques croisés sur le changement global et le développement - Langue, environnement, culture : Actes du Colloque international de Ouagadougou (8-10 mars 2012), Sciencesconf.org, 165 - 200.

13. Chimi Chiadjeu, O., Y. Gratton \& St-Hilaire, A. (2016). Analyse multivariée des paramètres morphologiques des lacs de la province du Québec. Rapport No R1684, INRS-ETE, Québec (QC), 30 p.

14. Dossou, M. E., Houessou, G. L., Lougbegnon, O. T., Tente, A. H. B. \& Codjia, J. T. C. (2012). Etude ethnobotanique des ressources forestières ligneuses de la forêt marécâgeuse d'Agonvè et terroirs connexes au bénin. Tropicultura, 30 (1): 41 - 48.

15. Dro, B., Soro, D., Koné, M. W., Bakayoko, A. \& Kamanzi, K. (2013). Valorisation de l'abondance de plantes médicinales utilisées en médecine traditionnelle dans le Nord de la Côte d'Ivoire. Journal of Animal \& Plant Sciences, 17 (3) : 2631 - 2646.

16. Dubut, O. (2012). Les beurres : Karité (Butyropermum parkii), cacao (Theobroma cacao), kokun (Garcinia indica) et illipé (Shorea stenoptera). Thèse de Doctorat, Université de Nantes, 128 p. 
17. Faye, E. (2010). Diagnostic partiel de la flore et de la végétation des Niayes et du Bassin arachidier au Sénégal : application de méthodes floristique, phytosociologique, ethnobotanique et cartographique. Thèse de Doctorat, Université Libre de Bruxelles, 266 p.

18. Ganaba, S., Ouadba, J-M. \& Bognounou, O. (2004). Plantes de construction d'habitations en région sahélienne. Bois et forêts des tropiques, 282 (4) : 11 - 17.

19. Gauze, T. K. M. \& Kanga, K. M-J. (2016). Problématique du zonage dans la gestion de la Réserve de Biosphère de la Comoé en Côte d'Ivoire. International Journal of Innovation and Applied Studies, 16 (2): 293 - 303.

20. Géhu, J. M. \& Géhu, J. (1980). Essai d'objection de l'évaluation biologique des milieux naturels. Exemples littoraux. In Géhu J.M. (ed). Séminaire de Phytosociologie Appliquée. Amicale Francophone de Phytociologie, Metz : 75-94.

21. Gormo, J. \& Nizesete, B. D. (2013). Des végétaux et leurs usages chez les peuples du Nord-Cameroun : sélection et mode d'emploi du XIXe au XXe siècle. História, Ciências, Saúde - Manguinhos, Rio de Janeiro, 20 (2) : 587 - 607.

22. Grangé, D., \& Lebart, L. (1993). Traitements Statistiques des Enquêtes. Dunod : Paris, 255 p.

23. Kambiré, B. (2010). L'agriculture vivrière du Nord-Est ivoirien en "régression" : un danger pour les centres urbains ivoiriens. Revue de Géographie Tropicale et d'Environnement, $2: 40$ - 53.

24. Kouakou, Y. B., Malan, D. F., Litta, A. L., Kouassi, K. G. \& Bakayoko, A. (2018). Plantes indicatrices des terres fertiles et de changement de saisons chez les Koulango et les Lobi riverains du Parc national de la Comoé, Nord-Est de la Côte d'Ivoire. Afrique SCIENCE, 14 (6) : $234-246$.

25. Kouamé, N. M. T., Gnahoua, G. M., Kouassi, K. E. \& Traoré, D. (2008). Plantes alimentaires spontanées de la région du Fromager (Centre-Ouest de la Côte d'Ivoire) : flore, habitats et organes consommés. Sciences \& Nature, $5: 61-70$.

26. Koulibaly, A. V. (2008). Caractéristiques de la végétation et dynamique de la régénération, sous l'influence de l'utilisation des terres, dans des mosaïques forêts-savanes, des régions de la réserve de Lamto et du parc national de la Comoé, en Côte d'Ivoire. Thèse, Université de Cocody Abidjan, $150 \mathrm{p}$.

27. Malan, D. F. (2008). Utilisations traditionnelles des plantes et perspective de cogestion des aires protégées de Côte d'Ivoire : cas du Parc National des Iles Ehotilé (littoral est de la Côte d'Ivoire), Thèse de Doctorat, Université d'Abobo-Adjamé, 194 p. 
28. Martin, G. L. (1995). Ethnobotany. A Methods Manuel. Royal Botanic Gardens, Kew, UK, Chapman \& Hall: London; 268 p.

29. Nonviho, G. (2015). Valorisation chimique de la biomasse oléagineuse d'origine Béninoise : Lophira lanceolata et Carapa procera. Thèse de Doctorat, Université d'Abomey-Calavi, $196 \mathrm{P}$.

30. Ouattara, M. (2014). Développement et mise en place d'une méthode de classification multi-bloc. Application aux données de l'OQAI. Thèse de Doctorat, Ecole Doctorale informatique, télécommunication et Electronique de Paris, 199 p.

31. Ouattara, N. D., Gaille, E., Stauffer, F. W. \& Bakayoko, A. (2016). Diversité floristique et ethnobotanique des plantes sauvages comestibles dans le Département de Bondoukou (Nord- Est de la Côte d'Ivoire). Journal of Applied Biosciences, 98 : 9284 - 9300.

32. OIPR (2015). Plan d'aménagement et de gestion du Parc National de la Comoé, site du patrimoine mondial et d'une Réserve de biosphère. Office Ivoirien des Parcs et Réserves, 116p.

33. Philips, O. \& Gentry, A. H. (1993). The useful plants of Tambopata, Peru. II Statistical hypothesis tests with a new quantitative technique. Economic Botany, 47 (1): 33 - 43.

34. Piba, S. C., Tra Bi, F. H., Konan, D., Bitignon, B. G. A. \& Bakayoko, A. (2015). Inventaire et disponibilité des plantes médicinales dans la forêt classée de Yapo-Abbé, en Côte D’ivoire. European Scientific Journal, 11 (24) : 161-181.

35. Rabiou, H., Diouf, A., Bationo, B. A., Segla, K. N., Adjonou, K., Kokutse, A. D., Radji, R., Kokou, K., Mahamane, A. \& Saadou, M. (2015). Structure des peuplements naturels de Pterocarpus erinaceus Poir. dans le domaine soudanien, au Niger et au Burkina Faso. Bois et forêts des tropiques, 325 (3) : $71-83$.

36. Schrauf, R.W. \& Sanchez, J. (2008). Using freelisting to identify access and characterize âge differences in shared cultural domains. Psychological Sciences and Social Sciences, 63 : 385 - 393.

37. Shango, M. (2010). Revue Nationale sur les Produits Forestiers non Ligneux (PFNL). Cas de la République Démocratique du Congo. Establishment of Forestry Research Network for ACP Countries (FORENET), 89p.

38. Soro, B., Sokouri, D. P., Dayo, G. K., N'Guetta, A. S. P. \& Gnaoré, C. V. (2015). Caractérisation des bovins de race Baoulé dans le "Pays Lobi" de Côte d'Ivoire : rôles socio-économiques, modes d'élevage et contraintes de production. Tropicultura, 33 (2) : 111 - 124.

39. Thiombiano, D. N. E., Lamien, N., Dibong, S. D. \& Boussim, I. J. (2010). Etat des peuplements des espèces ligneuses de soudure des 
communes rurales de Pobé-Mengao et de Nobéré (Burkina Faso). Journal of Animal \& Plant Sciences, 9 (1): 1104 - 1116.

40. Thomas E., Vandebroek I., Sanca S. \& Van Damme P., 2009. Cultural significance of medicinal plant families and species among Quechua farmers in Apillapampa, Bolivia. Journal of Ethnopharmacology, 122: $60-67$.

41. Thompson, E. C. \& Zhang, J. (2006). Comparative Cultural Salience: Measures Using Free-List Data. Field Methods, 18 (4): 398 - 412.

42. Tiébré, M. S., Ouattara, D., Vroh Bi, T. A., Gnagbo, A. \& N'Guessan, K. E. (2016). Diversité floristique et disponibilité des plantes utilitaires en zone soudanienne de la Côte d'Ivoire. Journal of Applied Biosciences, 102: 9699 - 9707.

43. Tiétiambou, F. R. S., Lykke, A. M., Korbéogo, G., Thiombiano, A. \& Ouédraogo, A. (2016). Perceptions et savoirs locaux sur les espèces oléagineuses locales dans le Kénédougou, Burkina Faso. Bois et forêts des tropiques, 327 (1): 39 - 50.

44. Vroh Bi, T. A., Ouattara, D. \& Kpangui, K. B. (2014). Disponibilité des espèces végétales spontanées à usage traditionnel dans la localité d'Agbaou, Centre-ouest de la Côte d'Ivoire. Journal of Applied Biosciences, 76: 6386 - 6396.

45. Ward, J. Jr. (1963). « Hierarchical grouping to optimize an objective function. » Journal of the American statistical association, vol. 58 (1301): 236 - 244.

46. Zerbo, P., Millogo-Rasolodimby, J., Guinko, S. \& Van Damme, P. (2014). Impact des tradipraticiens de santé dans la gestion durable des plantes médicinales au Burkina Faso : cas du Pays San. Revue CAMES Série Pharmacologie Médicinale Traditionnelle Africaine, 17 (1) : 59 - 66. 


\begin{tabular}{|c|c|c|c|c|c|c|}
\hline \multirow[t]{2}{*}{ Plantes } & \multirow[t]{2}{*}{ Ethnie } & \multicolumn{3}{|c|}{$\begin{array}{l}\text { ANNEXE } \\
\text { llisées dans la catégorie artisanale }\end{array}$} & \multicolumn{2}{|c|}{ Objets Domestiques } \\
\hline & & $\begin{array}{c}\text { Type de } \\
\text { construction }\end{array}$ & $\begin{array}{l}\text { Partie de la } \\
\text { construction }\end{array}$ & Ustensile & Aratoire & Mobilier \\
\hline Acacia dudgeonii & Koulango & Hangar, grenier & Traverse & Pilon & Manche de houe & \\
\hline Acacia nilotica & Koulango & & & & Manche de houe & \\
\hline $\begin{array}{c}\text { Anogeissus } \\
\text { leiocarpus }\end{array}$ & Koulango & Maison & Armature & Pilon & Manche de houe & \\
\hline Adansonia digitata & Koulango, Lobi & & & & & Tabourets \\
\hline Azadirachta indica & Koulango, Lobi & Hangar, grenier & Traverse & & & Chaises \\
\hline Bambusa vulgaris & Koulango & Maison, hangar & Armature, traverse & & & $\begin{array}{l}\text { Pointes de } \\
\text { chaises }\end{array}$ \\
\hline Bauhinia thonningii & Koulango, Lobi & Maison, hangar & Corde & & Manche de houe & \\
\hline Berlinia confusa & Koulango & & & $\begin{array}{l}\text { Mortier, } \\
\text { pilon }\end{array}$ & Manche de houe & \\
\hline Berlinia grandiflora & Koulango & & & Mortier & Manche de houe & \\
\hline Blighia sapida & Koulango, Lobi & & & & Manche de houe & \\
\hline Bombax costatum & Koulango, Lobi & & & Spatule & & Tabouret \\
\hline Borassus aethiopum & Koulango, Lobi & Hangar & Toiture & Eventail & & \\
\hline Bridelia ferruginea & Koulango, Lobi & & & Pilon & & \\
\hline Burkea africana & Koulango, Lobi & Hangar, grenier & Armature, traverse & Pilon & & \\
\hline Ceiba pentandra & Koulango, Lobi & & & & & Tabourets \\
\hline Cissus populnea & Koulango & $\begin{array}{l}\text { Maison, hangar, } \\
\text { grenier }\end{array}$ & Corde & & & \\
\hline Clausena anisata & Koulango & & & & & $\begin{array}{l}\text { Pointes de } \\
\text { chaises }\end{array}$ \\
\hline Cola gigantea & Koulango & Hangar & Corde & & & \\
\hline Cola laurifolia & Koulango & Maison & Poutre, traverse & & & \\
\hline Combretum molle & Koulango & Hangar, grenier & Traverse & & Manche de houe & \\
\hline Combretum collinum & Koulango & & & & Manche de houe & \\
\hline
\end{tabular}




\begin{tabular}{ccc} 
Cordia myxa & Koulango & Corde \\
grenier & Kaison, hangar, \\
\hline
\end{tabular}

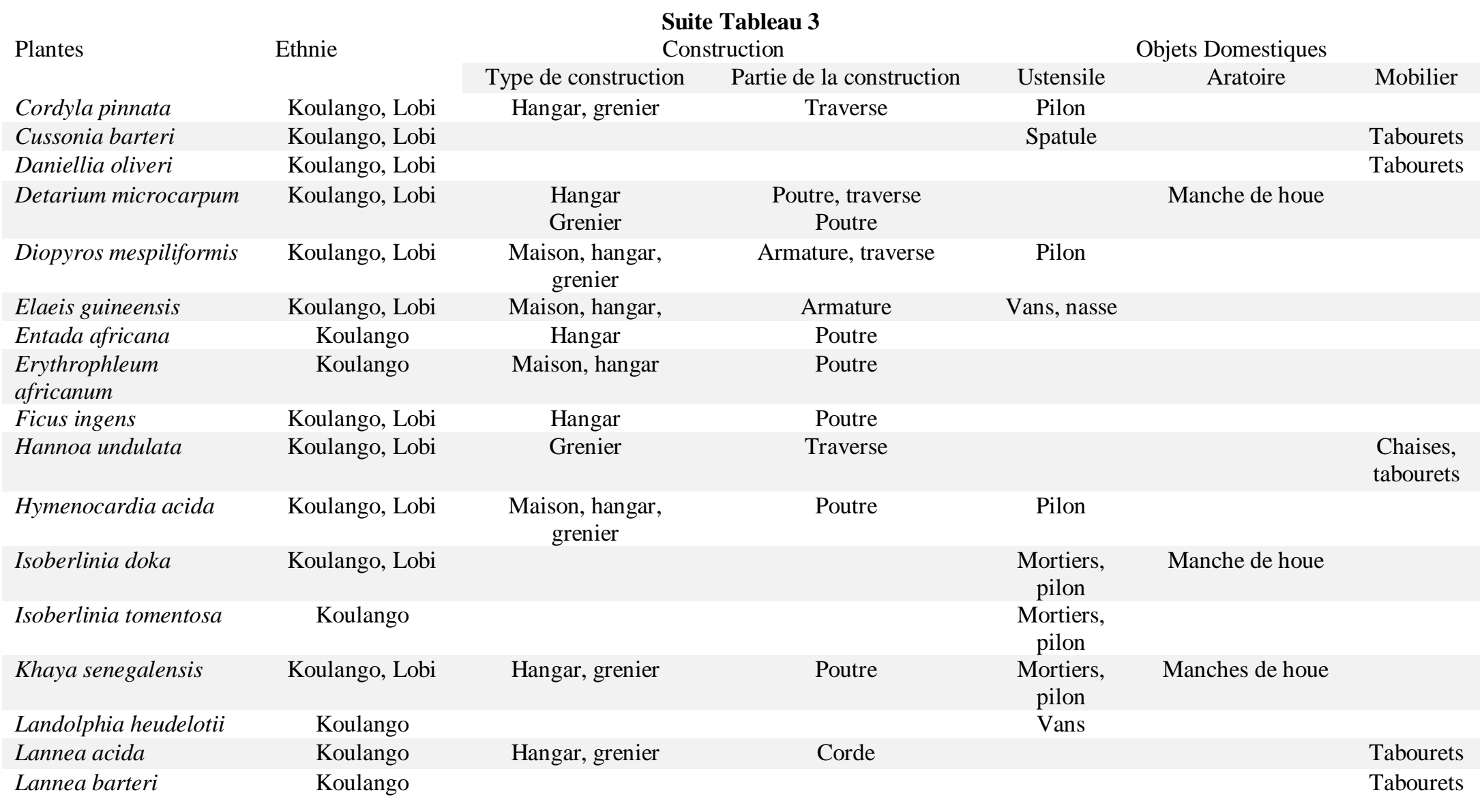




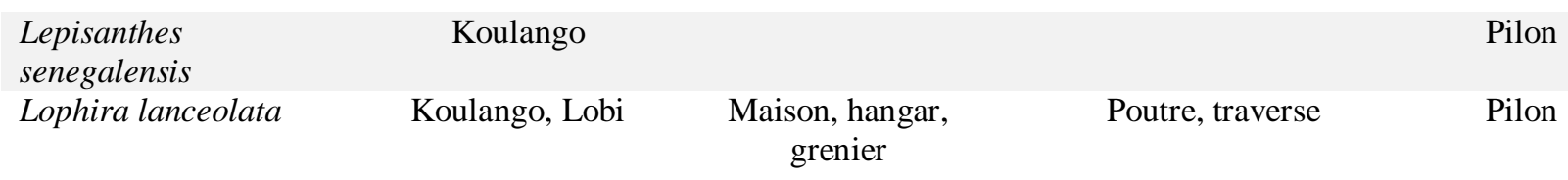

\begin{tabular}{|c|c|c|c|c|c|c|}
\hline \multicolumn{7}{|c|}{ Suite Tableau 3} \\
\hline \multirow[t]{2}{*}{ Plantes } & \multirow[t]{2}{*}{ Ethnie } & Construction & \multicolumn{4}{|c|}{ Objets Domestiques } \\
\hline & & $\begin{array}{c}\text { Type de } \\
\text { construction }\end{array}$ & $\begin{array}{l}\text { Partie de la } \\
\text { construction }\end{array}$ & Ustensile & Aratoire & Mobilier \\
\hline Manilkara multinervis & Koulango, Lobi & Hangar, grenier & Traverse & $\begin{array}{l}\text { Mortier, } \\
\text { pilon }\end{array}$ & & \\
\hline Milicia excelsa & Koulango & & & Mortier & & \\
\hline Mitragyna inermis & Koulango, Lobi & Hangar, grenier & Traverse & & & Chaise \\
\hline Monotes kerstingii & Koulango & Hangar, grenier & Traverse & Pilon & Manche de houe & \\
\hline Parinari curatellifolia & Koulango, Lobi & Hangar & Poutre & & Manche de houe & \\
\hline Parkia biglobosa & Koulango, Lobi & & & & Manche de houe & \\
\hline Paullinia pinnata & Koulango, Lobi & Hangar, grenier & Corde & & & \\
\hline Pericopsis laxiflora & Koulango, Lobi & Hangar, grenier & Poutre & & Manche de houe & \\
\hline Phoenix reclinata & Koulango, Lobi & Maison & Traverse & & & \\
\hline Prosopis africana & Koulango & Hangar, grenier & Traverse & & Manche de houe & \\
\hline $\begin{array}{l}\text { Pseudocedrela } \\
\text { kotschyi }\end{array}$ & Koulango, Lobi & $\begin{array}{l}\text { Maison, hangar, } \\
\text { grenier }\end{array}$ & Traverse & $\begin{array}{l}\text { Mortier, } \\
\text { pilon }\end{array}$ & & \\
\hline Pterocarpus erinaceus & Koulango, Lobi & Grenier & Poutre & Pilon & Manche de houe & \\
\hline Raphia sudanica & Koulango, Lobi & $\begin{array}{l}\text { Maison, } \\
\text { grenier }\end{array}$ & Armature & Vans & & $\begin{array}{l}\text { Chaises, lits, } \\
\text { tables et } \\
\text { tabourets }\end{array}$ \\
\hline Saba senegalensis & Koulango & Hangar & Corde & Vans & & \\
\hline $\begin{array}{c}\text { Sarcocephalus } \\
\text { latifolius }\end{array}$ & Koulango, Lobi & Hangar, grenier & Corde & & & \\
\hline Plantes & Ethnie & $\begin{array}{r}\text { Suite } \\
\text { Construc }\end{array}$ & $\begin{array}{l}\text { t fin Tableau } 3 \\
\text { ion }\end{array}$ & & ts Domestiques & \\
\hline
\end{tabular}




\begin{tabular}{|c|c|c|c|c|c|c|}
\hline & & $\begin{array}{c}\text { Type de } \\
\text { construction }\end{array}$ & $\begin{array}{l}\text { Partie de la } \\
\text { construction }\end{array}$ & Ustensile & Aratoires & Mobiliers \\
\hline Syzygium guineense & Koulango, Lobi & Hangar & Poutre & & & \\
\hline Tamarindus indica & Koulango, Lobi & & & Pilon & & \\
\hline $\begin{array}{c}\text { Terminalia } \\
\text { avicennioides }\end{array}$ & Koulango, Lobi & Hangar & Poutre & & & \\
\hline $\begin{array}{l}\text { Terminalia } \\
\text { macroptera }\end{array}$ & Koulango, Lobi & Hangar & Poutre & & & \\
\hline Trichilia emetica & Koulango & Hangar & Poutre & & & \\
\hline Uapaca togoensis & Koulango, Lobi & Hangar & Poutre & & & \\
\hline Uvaria chamae & Koulango, Lobi & & & Vans & & \\
\hline Vitellaria paradoxa & Koulango, Lobi & $\begin{array}{c}\text { Maison, hangar, } \\
\text { grenier }\end{array}$ & Poutre & Mortier, pilon & Manche de houe & Tabourets \\
\hline Vitex doniana & Koulango, Lobi & Hangar, grenier & Poutre & & & \\
\hline $\begin{array}{c}\text { Zanthoxylum } \\
\text { zanthoxyloides }\end{array}$ & Koulango, Lobi & & & & Manche de houe & \\
\hline
\end{tabular}

